\title{
Circuit
}

Musiques contemporaines

\section{Chute/Parachute de Michel Gonneville : pensée sérielle, écriture postmoderne (ou l'inverse)}

\section{Nicolas Gilbert}

Volume 19, numéro 3, 2009

Pionniers canadiens de la lutherie électronique

URI : https://id.erudit.org/iderudit/038262ar

DOI : https://doi.org/10.7202/038262ar

Aller au sommaire du numéro

Éditeur(s)

Les Presses de l'Université de Montréal

ISSN

1183-1693 (imprimé)

1488-9692 (numérique)

Découvrir la revue

Citer cet article

Gilbert, N. (2009). Chute/Parachute de Michel Gonneville : pensée sérielle, écriture postmoderne (ou l'inverse). Circuit, 19(3), 91-108.

https://doi.org/10.7202/038262ar
Résumé de l'article

Cet article est un texte analytique sur l'oeuvre Chute/Parachute du compositeur québécois Michel Gonneville. Après une brève introduction concernant les sources extramusicales de l'oeuvre, on expose ses enjeux généraux ainsi que ses principaux matériaux. On donne ensuite une vue d'ensemble de la structure formelle avant de passer à un examen plus poussé de la question du contrepoint dans l'oeuvre. Le texte se termine sur quelques remarques concernant le rapport entre la structure et la perception dans le travail de Gonneville. 
CAHIER D'ANALYSE

\title{
Chute/Parachute de Michel \\ Gonneville: pensée sérielle, écriture postmoderne (ou l'inverse)
}

Nicolas Gilbert

\begin{abstract}
Le tuyau auquel il devait adapter le chapiteau se trouvait au milieu du toit. Gervaise, tranquillisée, continuait à sourire en suivant ses mouvements. Nana, amusée tout d'un coup par la vue de son père, tapait dans ses petites mains. Elle s'était assise sur le trottoir, pour mieux voir là-haut.

«Papa! papa! criait-elle de toute sa force; papa! Regarde donc! »

Le zingueur voulut se pencher, mais son pied glissa. Alors, brusquement, bêtement, comme un chat dont les pattes s'embrouillent, il roula, il descendit la pente légère de la toiture, sans pouvoir se rattraper.
\end{abstract}

«Nom de Dieu!» dit-il d'une voix étouffée.

Et il tomba. Son corps décrivit une courbe molle, tourna deux fois sur lui-même, vint s'écraser au milieu de la rue avec le coup sourd d'un paquet de linge jeté de haut.

Émile Zola, L'Assommoir'

1. Zola (2000), p. 161-162.

En commençant à réfléchir à cet article analytique sur Chute/Parachute, je ne pouvais pas ne pas penser à la chute de Coupeau, dans L'Assommoir, l'une des chutes les plus célèbres de la littérature. Clef de voûte du roman, mais décrite sans la moindre trace de pathos, cette chute annonce la dégringolade sociale de Coupeau et de sa femme Gervaise. L'Assommoir est le récit d'une brève et modeste ascension, puis d'une longue et vertigineuse chute. J'ai pensé à L'assommoir parce que, oui, il y a bel et bien une chute dans et derrière Chute/Parachute, cette œuvre majeure de Michel Gonneville. Une chute qui, si elle n'a pas la dimension métaphorique de celle de Coupeau, a 
une réelle importance en tant que motif, comme dans l'œuvre de Zola. Et on pourrait dire la même chose d'une troisième chute, celle du personnage de Paul Auster, Sachs, dans Léviathan:

Deux personnes seulement ont effectivement vu Sachs tomber : Maria Turner, qui se tenait avec lui sur l'échelle de secours, et une certaine Agnès Darwin, qui lui fit par inadvertance perdre l'équilibre en trébuchant dans le dos de Maria. Il est incontestable que Sachs aurait pu se tuer. Compte tenu qu'il se trouvait à une hauteur de quatre étages, le contraire paraît tenir du miracle. Sans la corde à linge qui freina sa chute à un mètre cinquante du sol environ, il n'aurait jamais pu s'en tirer sans une incapacité permanente [...]. En fait, la corde cassa sous le poids de son corps et, au lieu de s'écraser la tête la première sur le ciment nu, il atterrit sur un fouillis moelleux de draps de bain, de couvertures et de serviettes. Non seulement Sachs survécut, il ressortit de l'accident relativement indemne. [...] Son corps a guéri, mais lui n’a plus jamais été le même. Comme si, en ces quelques secondes, Sachs avait tout perdu. Sa vie entière s'est éparpillée à mi-hauteur et, de ce moment à sa mort, quatre ans plus tard, il n'a jamais réussi à la reprendre en main. ${ }^{2}$

J'ai demandé à Gonneville de me parler de la chute qu'il a faite et qui est à l'origine du titre de l'œuvre. Le récit qu'il m'en a fait présente des parallèles frappants avec l'histoire de Sachs:

Pour envoyer quelques croûtes de pain aux oiseaux, en plein hiver, j'étais sorti en petites sandales chinoises avec semelles en plastique sur notre balcon glacé, et mon geste du bras m'a déséquilibré, et j'ai basculé par-dessus la balustrade. Et c'est la corde à linge qui m'a empêché de me retrouver au sol et m'a plutôt fait dévier vers le balcon du premier, où je me suis retrouvé sur mes deux pieds après avoir heurté brutalement la balustrade de ce balcon avec mon fémur gauche et mon menton, complètement sonné, les gencives en sang, dévisagé par les voisins d'en bas sortis sur leur balcon et éberlués, etc. J'ai eu des cauchemars de chutes pendant plusieurs années jusqu'à ce que j'aille en thérapie, où j'ai accepté de revivre la chute en imagination, en acceptant son terme possible: la mort. Or cette chute revécue en «rêve provoqué» s'est faite très lentement, et c'est ce qui a donné la première partie de Chute/Parachute. La chute de Gonneville n'a heureusement pas eu les conséquences tragiques de celle de Sachs, mais les deux chutes sont, à des degrés différents, imbibées de tragique. Sans doute les deux protagonistes, Sachs et Gonneville, ont-ils eu l'impression qu'on ne pouvait échapper à son destin et qu'une corde à linge ne pouvait, au fond, rien y changer; Sachs, tout particulièrement, avait l'impression qu'il aurait dû mourir. Et les deux accidents ont en commun d'être à l'origine d'une œuvre artistique: Chute/Parachute pour Gonneville et, pour Sachs, le projet artistico-terroriste de faire exploser toutes les reproductions de la statue de la Liberté se trouvant sur le sol américain. 
Mon intuition est qu'il ne faut pas se laisser prendre par l'affirmation de Gonneville selon laquelle son accident n'aurait eu d'impact que sur la première partie de Chute/Parachute. La chute, et plus particulièrement la chute «au ralenti» telle que revécue au cours de son processus de thérapie, me semble au contraire être un motif fondamental dans le travail de Gonneville. N'est-ce pas une autre chute au ralenti, ou plutôt, n'est-ce pas précisément la même chute qui est représentée par la baleine nageant jusqu'au fond de la mer dans Le cheminement de la baleine 3 , une œuvre composée dix ans après Chute/Parachute? Quoi qu'il en soit, la grande force de Chute/Parachute et de la musique de Gonneville en général se trouve à mon avis dans son travail des parcours, des mouvements directionnels, dont l'idée de chute est un exemple parfait. C'est ce qui la rend si attrayante aux auditeurs: la musique de Gonneville est constamment en mouvement. Il n’y a jamais de véritable statisme; les moments statiques en apparence sont en fait du mouvement en puissance, ce sont des moments de tension.

On parle souvent de Chute/Parachute comme de l'œuvre majeure de Gonneville. Ce fait justifie à lui seul d'en rendre l'analyse accessible à un large public. Mais au-delà de cela, les méthodes et stratégies compositionnelles de Gonneville sont à la fois si rigoureuses et si flexibles qu'elles peuvent servir de modèle aux jeunes compositeurs, dont je suis. Le travail analytique qui suit est largement basé sur la thèse de doctorat de Gonneville qui portait, entre autres, sur cette œuvre. Mon apport a été de mettre en lumière les éléments de l'analyse qui me semblaient les plus susceptibles de provoquer chez le lecteur des «illuminations» de différents types et de poser, ici et là, un regard extérieur sur le travail du compositeur.

\section{Enjeu général}

Chute/parachute est une œuvre d'environ treize minutes pour piano et bande. Bien qu'il s'agisse d'une œuvre mixte, l'approche compositionnelle privilégiée par le compositeur est véritablement instrumentale. C'est que la bande ne fait entendre que des sons à hauteurs déterminées produits, à l'origine, par un piano électronique microtonal. Cette bande est en fait l'enregistrement d'une «interprétation», au synthétiseur, d'une partition extrêmement complexe. Gonneville se place donc ici bien davantage dans le champ de la musique instrumentale utopique 4 que dans celui de la musique acousmatique.

L'un des enjeux importants de cette œuvre est la confrontation de deux mondes harmoniques: le monde harmonique tempéré du piano et le monde harmonique non tempéré du synthétiseur. On verra en effet plus bas que le travail harmonique est, à la base, spectral, et que le piano et le synthétiseur
3. Le cheminement de la baleine (1998) est une œuvre pour clarinette, ondes Martenot et ensemble de 18 instrumentistes.

\footnotetext{
4. Musique faisant usage de moyens mécaniques (souvent électroacoustiques) pour rendre des partitions trop complexes pour être interprétées en temps réel. On pense, par exemple, aux Études pour piano mécanique de Conlon Nancarrow.
} 
mettent systématiquement en relation les versions tempérée et naturelle d'un même spectre. Il résulte de cette confrontation un travail complexe sur les battements et les oscillations. C'est de ce travail que découle une bonne part de la richesse timbrale de l'œuvre.

\section{Les deux modes}

Chute/parachute est entièrement conçue, sur le plan des hauteurs, sur deux modes issus de la série des harmoniques naturels. Voici d'abord, pour référence, les trente-deux premiers partiels harmoniques d'un do grave.

FIGURE 1. Série harmonique

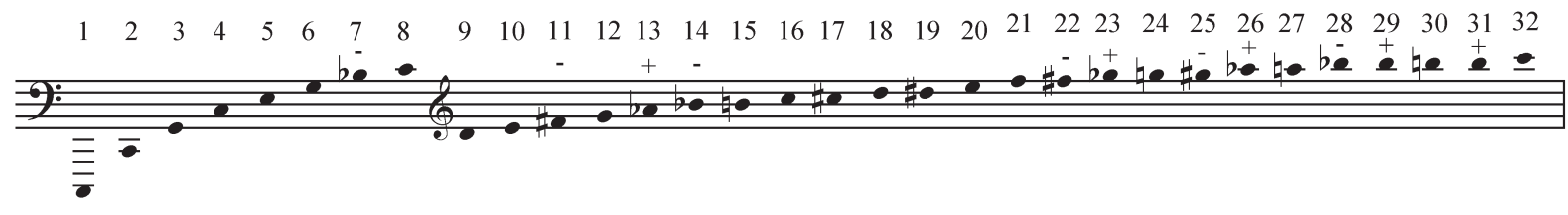

Observons maintenant les deux modes que Gonneville a extraits de ce spectre. En suivant l'habitude du compositeur, nous les identifierons par les numéros des partiels qu'ils contiennent.

FIGURE 2. Mode 8 à 15, forme originale

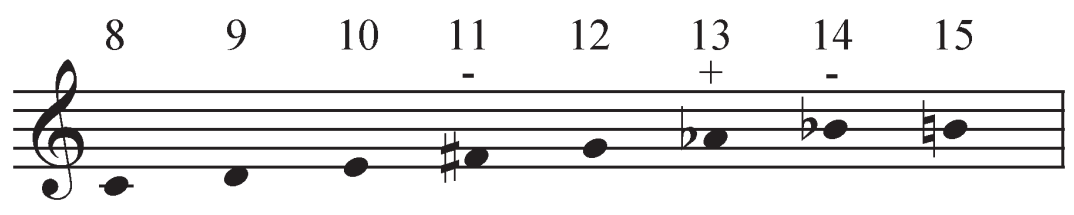

FIGURE 3. Mode 16 à 31, forme originale

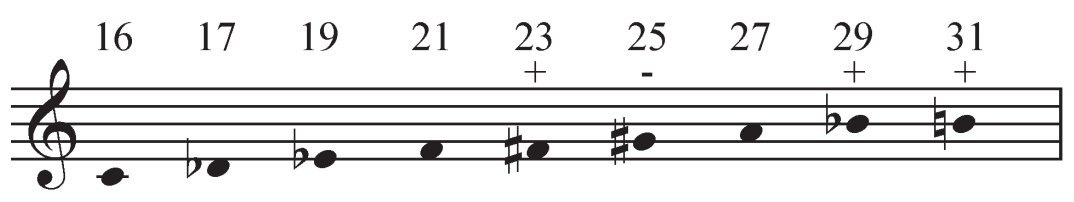


Les versions énoncées ci-dessus, qui débutent sur do et ont donc do pour «fondamentale», représentent ce que nous appellerons la forme originale des deux modes. Le compositeur a également utilisé différentes transpositions : chacun des deux modes a été transposé sur chacune des hauteurs constitutives de sa forme originale. On retrouve donc le mode 8 à 15 sur do (forme originale), ré, mi, fa dièse, sol, la bémol, si bémol et si, et le mode 16 à 31 sur do (forme originale), ré, mi, fa, fa dièse, sol dièse, la, si bémol, et si. Il est important de noter que le synthétiseur et le piano ont le même parcours modal, c'est-à-dire qu'à n'importe quel moment de l'œuvre, ils font entendre le même mode, dans la même transposition, en version tempérée pour le piano, et naturelle pour le synthétiseur. Soulignons également que, dans la partie de synthétiseur, les fondamentales des huit transpositions du mode correspondent non pas à la version tempérée de ces notes mais plutôt à leur version naturelle, ce qui produit souvent d'importantes frictions avec la partie de piano.

Le compositeur utilise à l'occasion un arrangement sériel du mode 8 à 15 .

FIGURE 4. Mode 8 à 15, arrangement sériel original

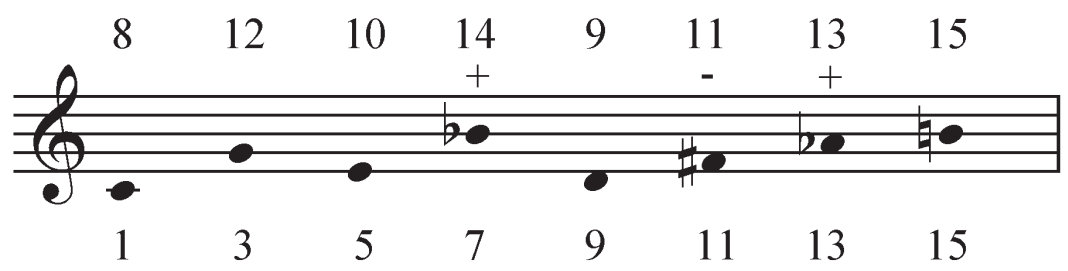

Cet arrangement sériel n'est nullement anodin puisque, sous cette forme, le mode présente, dans l'ordre, les harmoniques impairs de 1 à 15, compressés à l'intérieur d'une octave.

Par ailleurs, ces deux modes servent également à la détermination des durées. On verra que le compositeur détermine souvent la durée d'une note d'un mode en fonction de son numéro d'ordre dans la série des harmoniques. Par exemple, dans le mode 8 à 15, le son 8 pourra durer 8 unités de temps, le son 9, 9 unités de temps, et ainsi de suite. Il y a donc véritablement une pensée sérielle derrière le travail des durées. 


\section{Survol de la forme}

Le tableau synoptique suivant présente un résumé de la grande forme. Les titres donnés à chacune des parties sont ceux que propose le compositeur.

\begin{tabular}{|c|c|c|c|}
\hline A CHUTE/PARACHUTE & B ANTICHUTE (DANSE) & C CADENCE & D TRAMPOLINE \\
\hline mm. 1-82 & mm. 83-194 & mm. 195-217 & mm. 218-233 \\
\hline $\begin{array}{l}\text { Descente lente et } \\
\text { graduelle sur le mode } 16 \\
\text { à } 31 \text {. Contrepoint à quatre } \\
\text { parties. Le piano double et } \\
\text { ornemente le synthétiseur. } \\
\text { La fin de la partie mène vers } \\
\text { le mode } 8 \text { à } 15 \text {. }\end{array}$ & $\begin{array}{l}\text { Parcours en quatre sections } \\
\text { de plus en plus denses vers } \\
\text { un contrepoint final à quatre } \\
\text { parties sur le mode } 8 \text { à } 15 .\end{array}$ & $\begin{array}{l}\text { Partie de transition en } \\
\text { trois courtes sections. Un } \\
\text { matériau de type arpégé } \\
\text { est développé. Le piano } \\
\text { prédomine dans la } 1^{\text {re }} \text { et la } \\
3^{\text {e }} \text { section et dialogue avec le } \\
\text { synthétiseur dans la } 2^{\mathrm{e}} \text {. Sur le } \\
\text { mode } 8 \text { à } 15 .\end{array}$ & $\begin{array}{l}\text { Constitution progressive } \\
\text { d'un carillon harmonique au } \\
\text { synthétiseur. "Contrepoint } \\
\text { virtuel ", au piano, sur une } \\
\text { suite d'accords superposés, } \\
\text { également énoncés par la } \\
\text { bande. Sur le mode } 8 \text { à } 15 \text {, } \\
\text { arrangement sériel et } 16 \text { à 31, } \\
\text { progression en éventail. }\end{array}$ \\
\hline
\end{tabular}

5. Cette idée a été exploitée par Gonneville avec plus de systématisme et, peut-être, encore plus d'efficacité dans Le cheminement de la baleine.
La partie A nous fait parcourir le mode 16 à 31 de l'aigu vers le grave, sur un rythme lent et soutenu. Ce passage de l'aigu au grave constitue le principal élément de directionnalité de la partie. On retrouve également une très forte directionnalité sur le plan des intensités puisque autant le piano que le synthétiseur présentent un diminuendo graduel s'étendant sur pratiquement toute la partie. Dans cette partie, le piano se contente de doubler et d'ornementer certaines attaques du synthétiseur. Extrêmement discret au début de la partie, le piano est de plus en plus présent au fur et à mesure du déroulement de la partie, mais sans jamais vraiment occuper l'avant-plan.

L'attention est fortement sollicitée, dans cette première grande partie de l'œuvre, par une suite de notes pédales jouées par le synthétiseur. Ce sont ces pédales qui mènent en quelque sorte la descente du mode 16 à 31 et déterminent les transpositions du mode utilisées dans les voix supérieures. Une fois qu'ont été énoncés les neuf sons du mode dans sa forme originale, le son d'arrivée, la fondamentale do, est tenu jusqu'à la fin de la partie. Cette pédale de $d o$, qui s'étend de la mesure 49 à la mesure 82 (et même au-delà, dans la partie B) est très importante sur le plan de la perception. En plus d'instaurer un certain statisme harmonique et, en conséquence, une sorte de suspension temporelle, cette arrivée sur le do donne à l'auditeur l'impression d'avoir «touché le fond5». L'auditeur se met donc à attendre, plus ou moins consciemment, la remontée, qu'il sent nécessaire et imminente.

La partie B constitue justement cette remontée tant attendue. Cette partie présente une augmentation graduelle et continue de la densité, couplée à un 
long crescendo d'ensemble, qui aboutira à la portion la plus dense et la plus complexe de l'œuvre, la cinquième section de $\mathrm{B}$, que le compositeur appelle la «Danse». Nous examinerons cette section en détail plus loin. Disons pour le moment que les quatre sections qui y mènent sont des versions «trouées » de ce grand contrepoint et qu'elles s'en rapprochent de plus en plus. La partie $\mathrm{B}$ suit donc une courbe ascendante de tension qui mène au moment paroxystique de l'œuvre.

La partie C, la plus courte de l'œuvre, procède de la nécessité sentie par le compositeur d'installer une chute de tension à la suite du point culminant de la dernière section de la partie B. Au cours de cette partie, le piano joue pour la première fois presque seul par moments et occupe tout l'avant-plan. La partie est essentiellement constituée d'arpèges sur le mode 8 à 15, dans différentes transpositions. Les huit dernières mesures de la partie (mm. 210 à 217) présentent un accelerando et un crescendo qui produisent une augmentation de la tension et vont permettre de commencer la partie D dans un esprit similaire à ce qu'on retrouvait à la fin de la partie B.

Nous nous pencherons plus bas sur ce que donne à entendre le piano dans la partie D. Disons maintenant que cette dernière grande partie est plus monolithique que les précédentes en ceci qu'elle ne comporte qu'une seule section portée par une seule ligne directionnelle. Cette ligne directionnelle est, en fait, triple de nature : la partie de piano est de moins en moins complexe et de plus en plus lente et douce, les accords superposés joués par le synthétiseur sont de moins en moins touffus et de plus en plus courts, le carillon d'harmoniques entendu sur la bande comprend de plus en plus de sons et procède en crescendo. Toute cette partie est basée sur l'arrangement sériel du mode 8 à 15. L'œuvre se termine par l'énonciation, au synthétiseur, des harmoniques impairs d'un do grave jusqu'au $31^{\mathrm{e}}$, ce dernier harmonique se résolvant par glissando sur un do aigu, le $32^{\mathrm{e}}$ harmonique, qui assume donc ici pleinement sa fonction de tonique.

\section{Le contrepoint}

Du point de vue strictement technique, l'un des aspects les plus originaux de Chute/Parachute est sans contredit le travail contrapuntique. Gonneville a développé dans cette œuvre une technique d'écriture contrapuntique fortement formalisée qui découle directement de la tradition du sérialisme. En examinant deux sections contrapuntiques de l'œuvre, nous pourrons constater que la formalisation est ici toujours extrêmement «sensible », que ce qui en provient a systématiquement un impact direct sur la perception et qu'elle n'est donc pas une fin en elle-même pour le compositeur. 
La cinquième section de la partie B, la "Danse"

Le contrepoint à quatre voix sur lequel nous nous pencherons maintenant se trouve aux mesures 174 à 193, dans la partie de synthétiseur. Les quatre voix sont construites à partir du même matériau, le mode 8 à 15, dans son arrangement sériel qui, rappelons-le, équivaut aux huit premiers harmoniques impairs d'une fondamentale donnée. Les voix ont une registration fixe: elles sont placées à une octave de distance l'une de l'autre. La durée de chaque note est déterminée par le numéro d'ordre de celle-ci dans la série des harmoniques. Pour ce calcul des durées, Gonneville a associé les notes du mode aux harmoniques impairs de 1 à 15 plutôt qu'aux harmoniques 8 à 15 . Chaque voix a une unité de durée de base différente, soit, de l'aigu au grave, la triple croche pour la voix 1, la double croche pour la voix 2 , la croche pour la voix 3 et la noire pour la voix 4. Ces quelques règles nous permettent déjà de déduire toute l'armature du contrepoint. Cette armature peut être schématisée comme suit, les chiffres représentant à la fois le numéro d'harmonique et la durée de chaque son en nombre d'unités de temps (en fonction des correspondances données plus haut).

$\begin{array}{llllllllll}\text { Voix 1: } & 1 & 3 & 5 & 7 & 9 & 11 & 13 & 15 & \text { (entendu } 8 \text { fois) } \\ \text { Voix 2: } & 1 & 3 & 5 & 7 & 9 & 11 & 13 & 15 & \text { (entendu 4 fois) } \\ \text { Voix 3: } & 1 & 3 & 5 & 7 & 9 & 11 & 13 & 15 & \text { (entendu 2 fois) } \\ \text { Voix 4: } & 1 & 3 & 5 & 7 & 9 & 11 & 13 & 15 & \text { (entendu 1 fois) }\end{array}$

Afin d'y voir absolument clair, voici l'armature de la voix 4, pour tout le contrepoint (à noter que cette voix présente le mode sous sa forme originale, sur do).

FIGURE 5. Partie B, Section 5, armature de la voix 4 du contrepoint

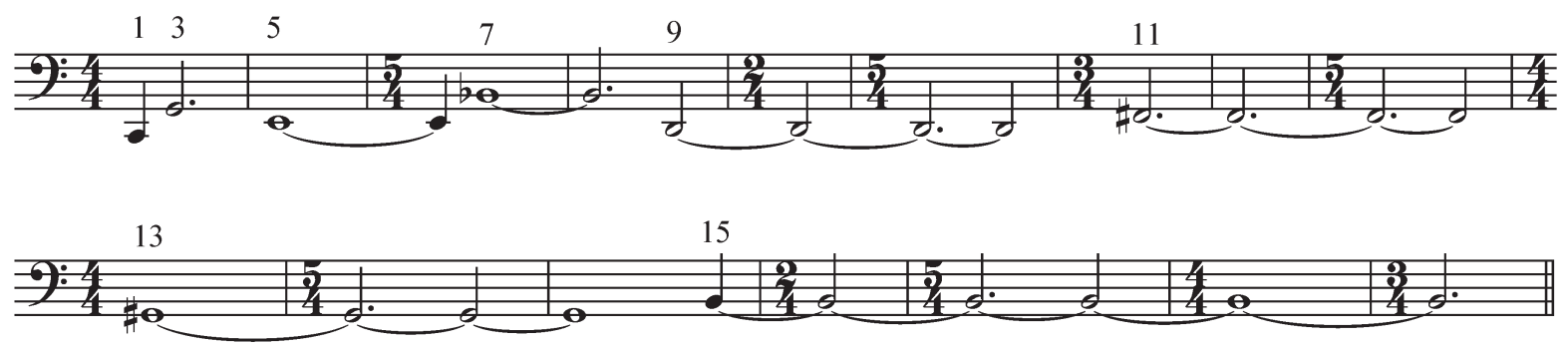

Cette armature est ensuite ornementée de façon relativement complexe. Chaque voix présente un mode d'ornementation distinct. Les quatre modes peuvent être résumés ainsi, toujours de l'aigu vers le grave: 
Voix 1: traits conjoints sur le mode

Voix 2: ornementation variée, broderies larges en expansion ou en contraction

Voix 3: broderies inférieures ou supérieures de la note structurelle

Voix 4: répétitions de la note structurelle

Il faut également noter qu'une fois écoulée la moitié de la durée de chaque note structurelle de l'armature (à la noire près et pour les durées plus longues qu'une noire), il se produit une modification dans l'ornementation. En reprenant la voix 4, cette fois-ci telle qu'elle se présente dans la partition, on constatera en effet que la vitesse de répétition de la note structurelle change une fois par note structurelle, vers le point milieu de sa durée totale.

FIGURE 6. Partie B, Section 5, voix 4 du contrepoint

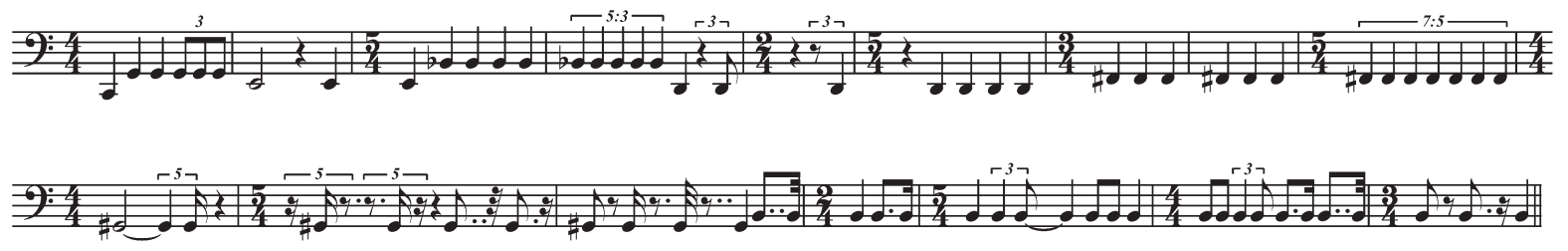

Nous avons vu que la voix 4 présente le mode en $d o$. Ce n'est pas toujours le cas des trois autres voix qui, elles, suivent un parcours modulatoire prédéfini. Ce parcours est en fait «contrôlé» par la voix 4, c'est-à-dire que chaque nouvelle note structurelle de la voix 4 devient la fondamentale des trois autres voix. Les trois voix supérieures sont donc en do pour un temps, en sol pour trois temps, en mi pour cinq temps, en si bémol pour sept temps, etc. À l'arrivée de chaque nouvelle note de la basse, les trois autres voix sont donc transposées, mais continuent leur cycle d'harmoniques impairs au point où elles en étaient, sans interruption.

Ces quelques explications mettant en relief les «règles du jeu » que s'est fixées le compositeur nous donnent une idée d'ensemble de la structure et des processus en jeu sous la surface de la partie de synthétiseur. La partie de piano suit une logique parallèle. De la mesure 174 jusqu'au $4^{\mathrm{e}}$ temps de la mesure 187 , le piano double successivement chacune des trois voix supérieures du contrepoint: voix 3 de 187 à $1821^{\mathrm{er}}$ temps, voix 2 de $1822^{\mathrm{e}}$ temps à $1853^{\mathrm{e}}$ temps, voix 1 de $1854^{\mathrm{e}}$ temps à $1874^{\mathrm{e}}$ temps. Il ne s'agit cependant pas de doublures simples puisque le piano ajoute de l'épaisseur harmonique à chacune des notes du contrepoint en construisant des accords sur le mode. 
Le piano marque également chaque transposition des trois voix supérieures du contrepoint (donc chaque nouvelle note structurelle de la voix 4) par un cluster. Tout ce système est brisé à partir du dernier temps de la mesure 187. À partir de ce moment, le piano fait entendre un tremolo frénétique qui aboutira sur un $f a$ dièse aigu, en unisson avec le synthétiseur. Ce fa dièse est la note commune à toutes les transpositions du mode et servira de porte d'entrée dans la partie C.

Voici, extrait de la partition, le début de ce grand contrepoint à quatre voix :

FIGURE 7. Chute/Parachute, mesures 173 à 184
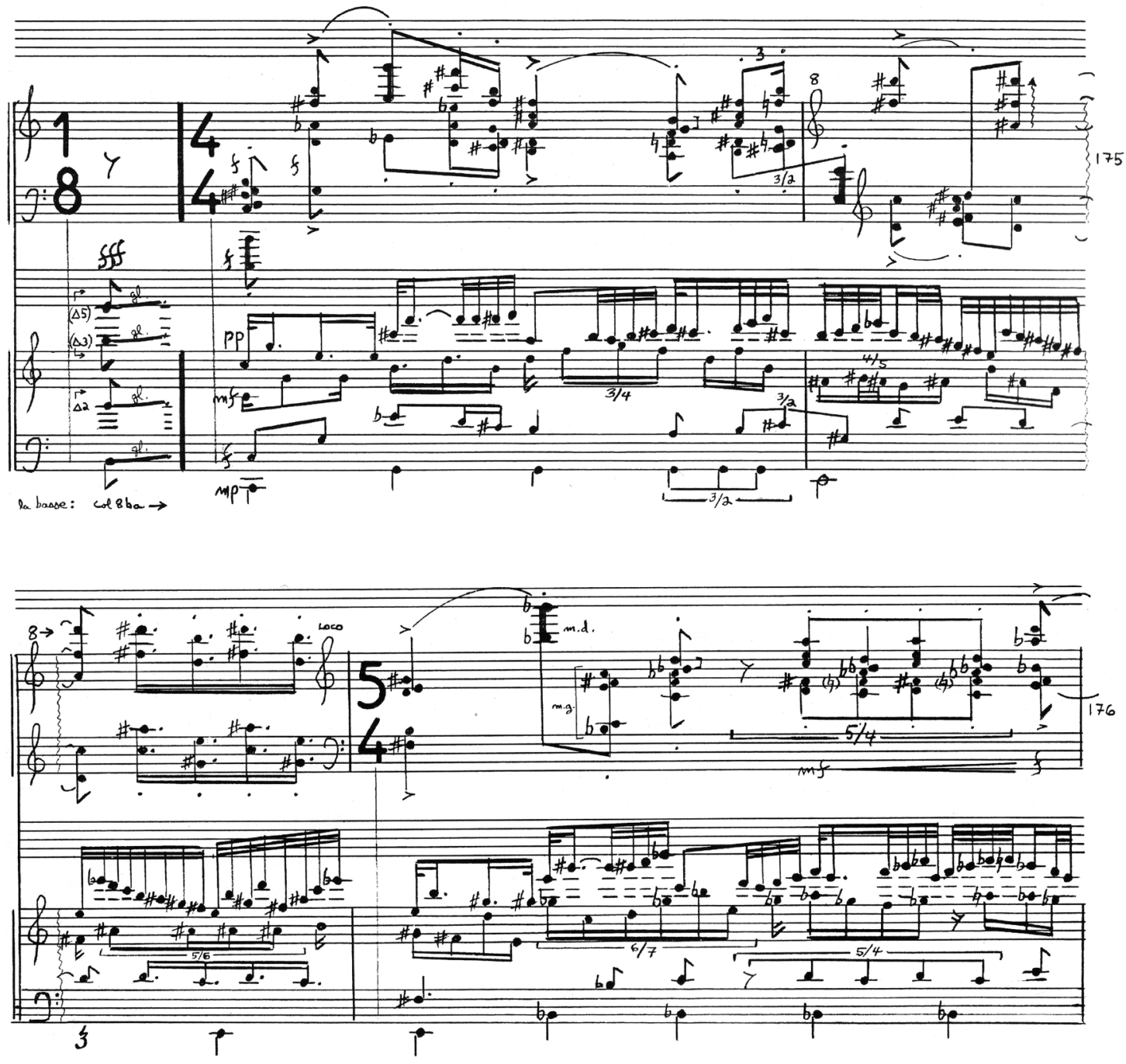

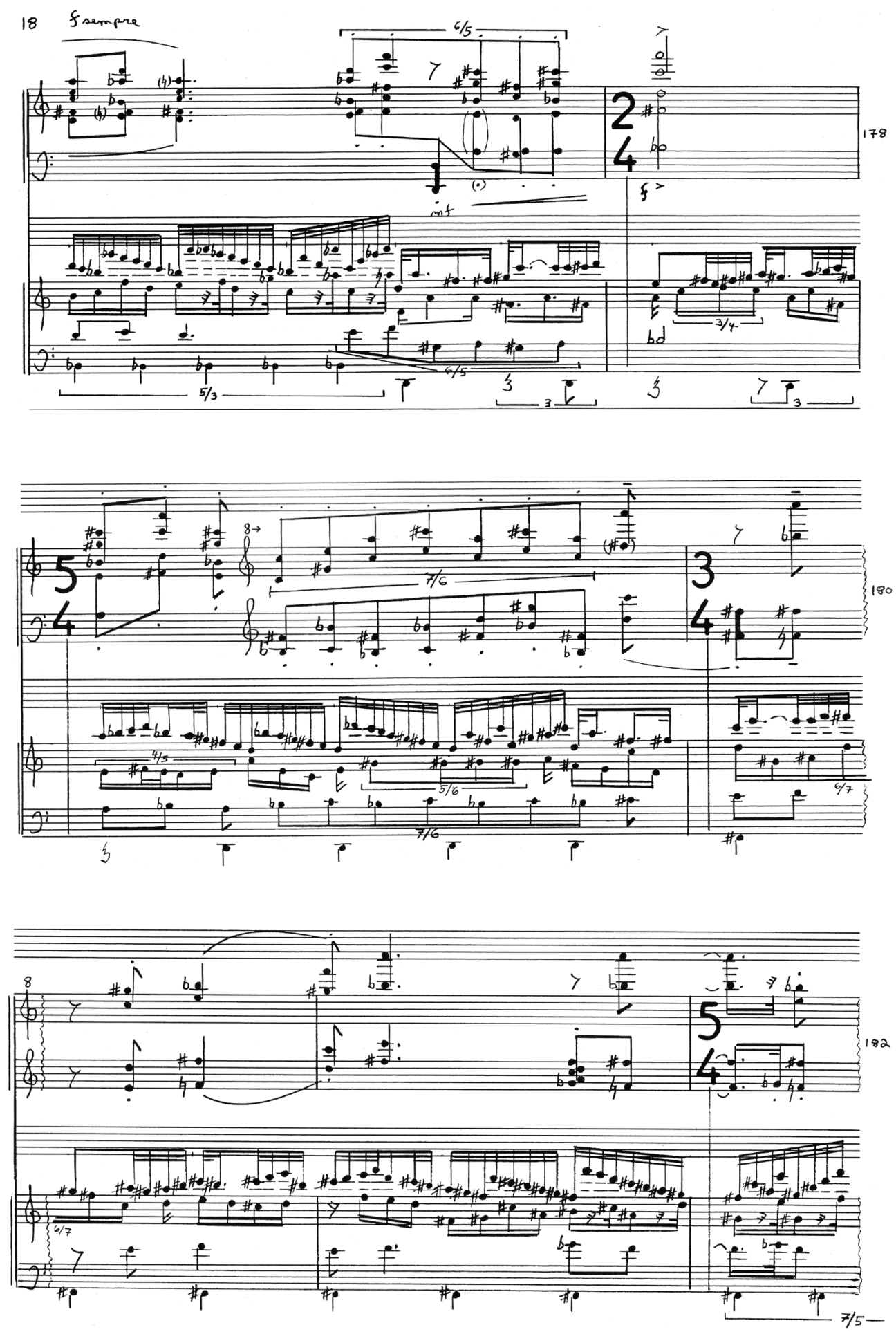

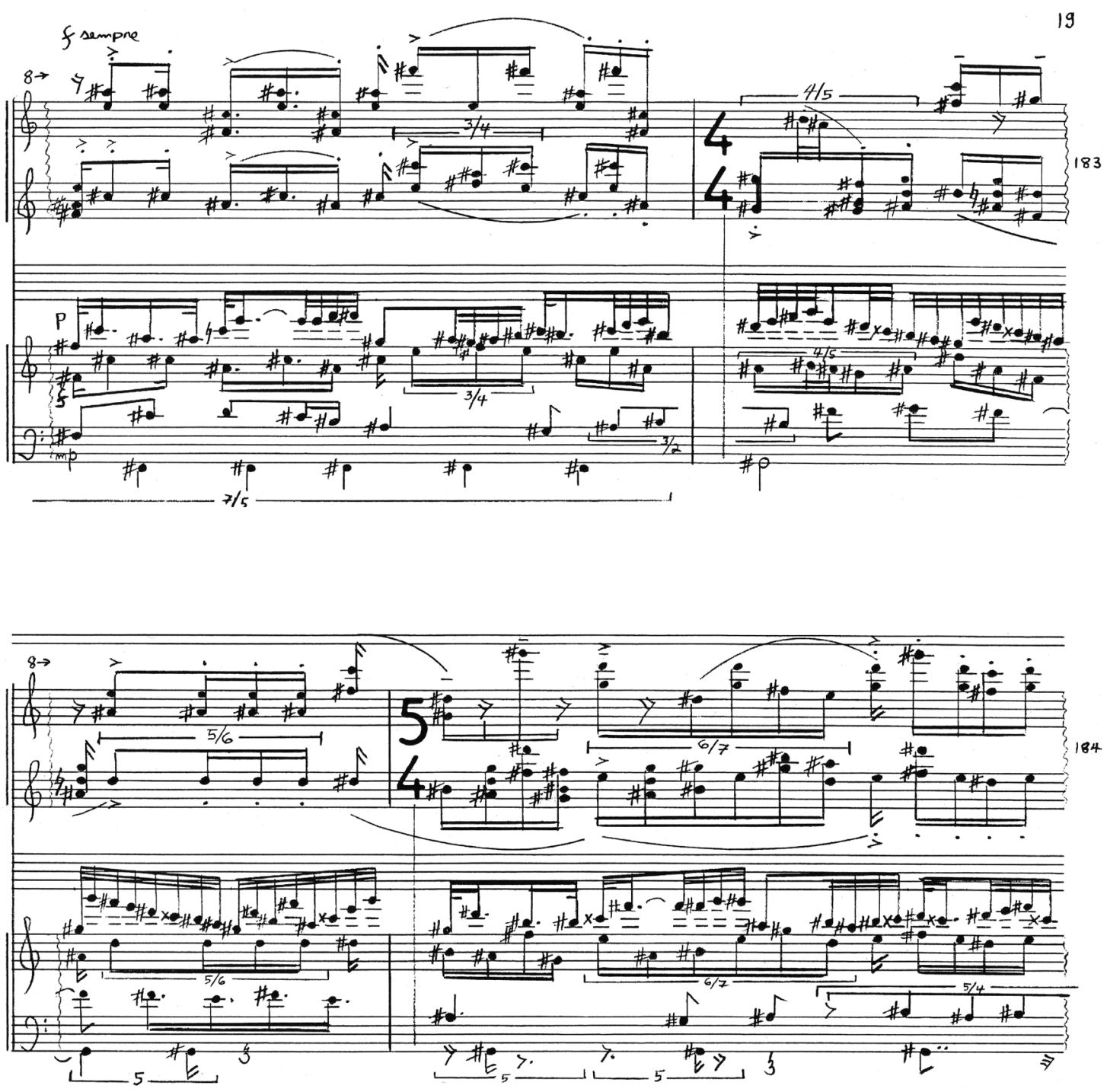

Le contrepoint "virtuel " de la partie D

La deuxième section de contrepoint sur laquelle nous nous pencherons occupe les mesures 218 à 233, dans la partie de piano. Ce contrepoint n'en est pas tout à fait un; il est «virtuel» en ceci que les quatre voix qui le composent ne sont pas aisément discernables et donnent plutôt l'impression de n'en former qu'une seule. L'écriture est donc ici contrapuntique davantage sur le plan poḯtique que sur le plan esthésique. Voici les deux premières mesures de cette section, telles que présentées dans la partition: 
FIGURE 8. Chute/Parachute, mesures 218 et 219

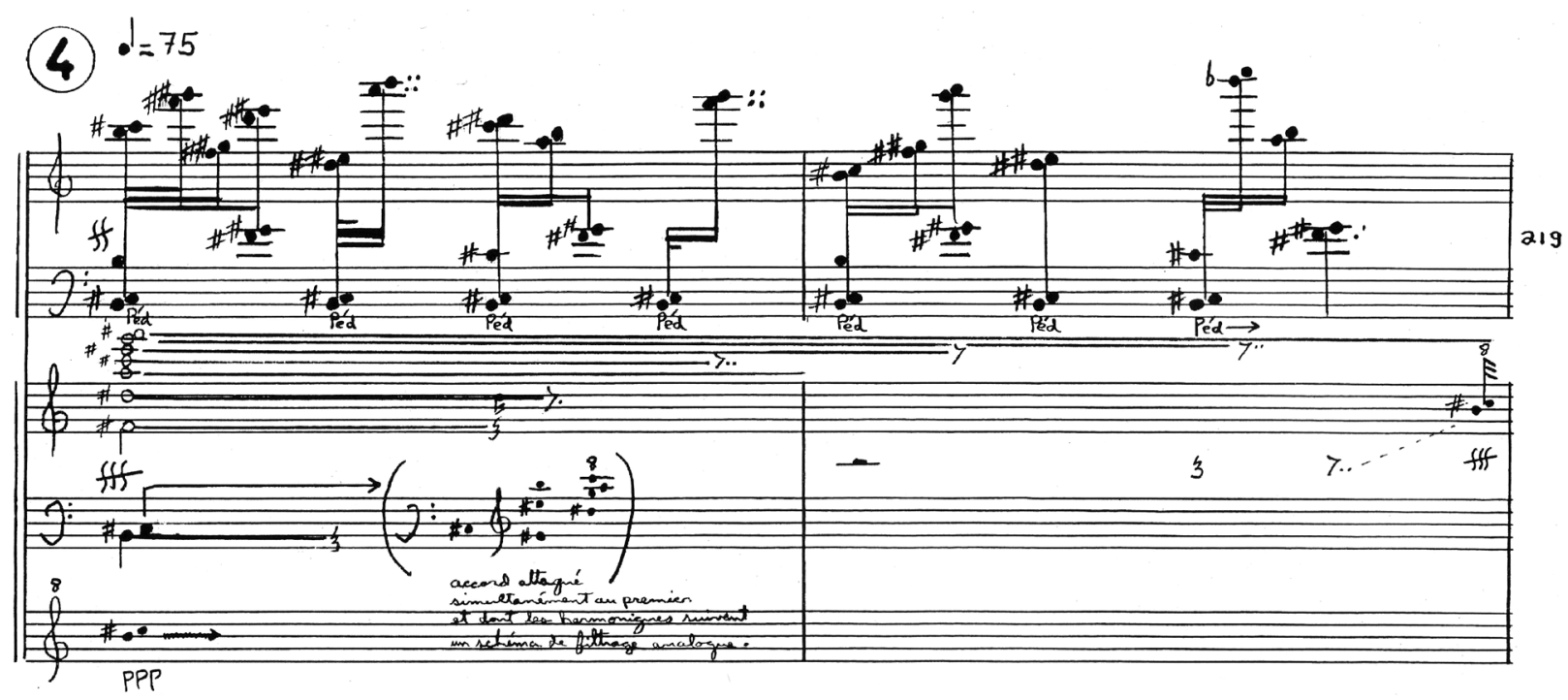

Observons maintenant le contrepoint à proprement parler, dont la partie de piano est la réduction:

FIGURE 9. Contrepoint virtuel, mesures 218 à 232

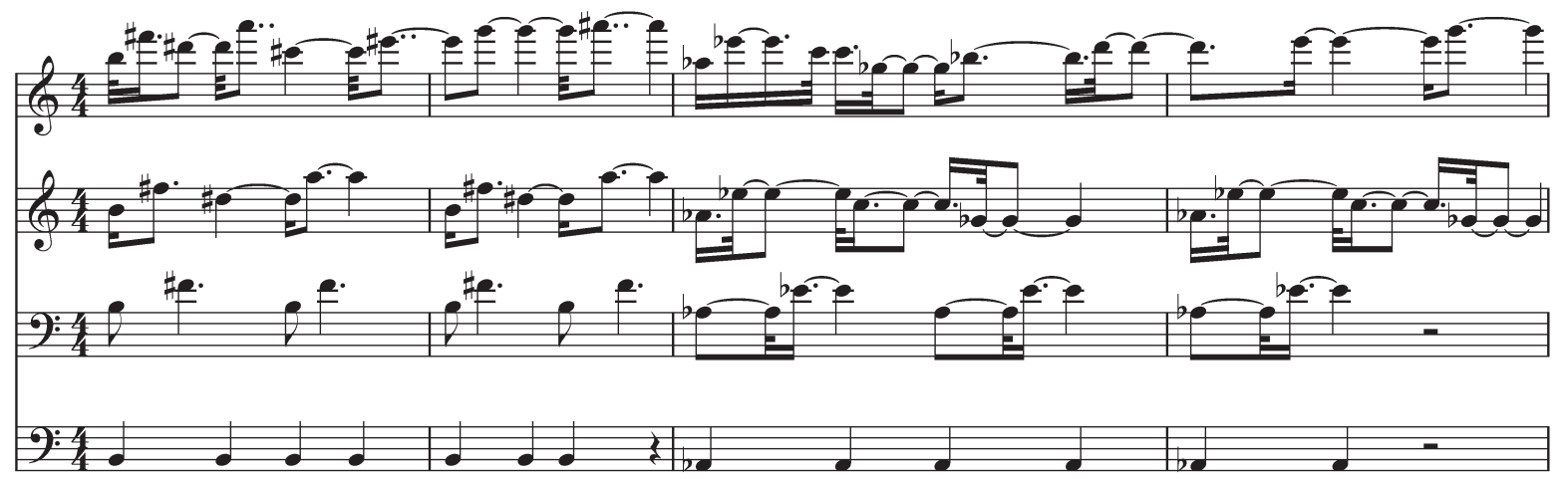



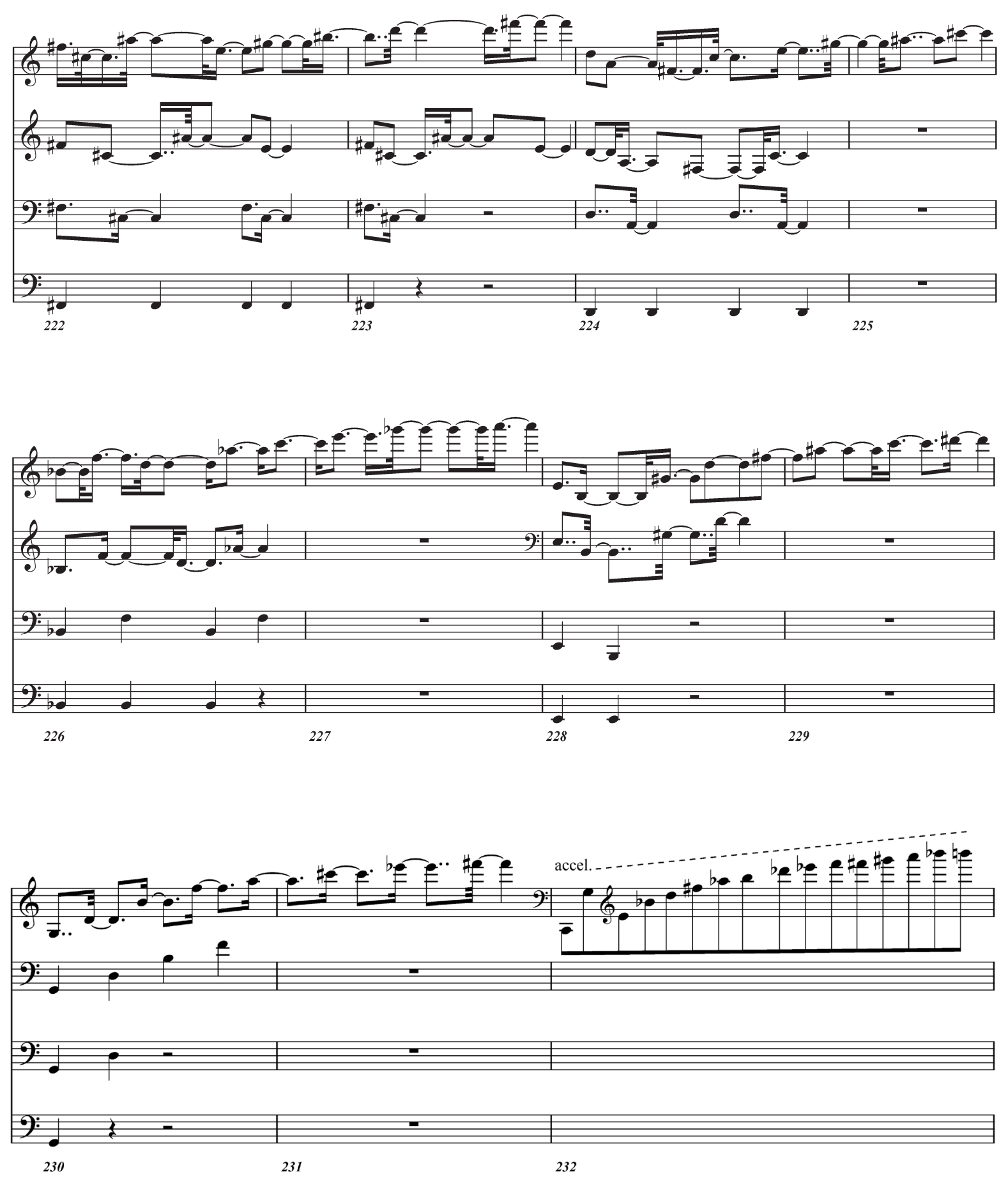
La technique compositionnelle est apparentée à ce qui a été utilisé pour la conception de l'armature du contrepoint final de la partie B. On constate que tout le contrepoint est divisé en segments de deux mesures, chaque segment présentant une nouvelle note dans la voix 4 . Ces notes sont celles du mode 8 à 15, dans son arrangement sériel rétrograde. Encore une fois, ces notes de basse sont autant de fondamentales contrôlant les transpositions des trois parties supérieures. Le schéma suivant donne la disposition de base des notes du mode, selon leur numéro d'harmonique, pour le premier segment de deux mesures.

$\begin{array}{lllllllll}\text { Voix 1: } & 1 & 3 & 5 & 7 & 9 & 11 & 13 & 15 \\ \text { Voix 2: } & 1 & 3 & 5 & 7 & 1 & 3 & 5 & 7 \\ \text { Voix 3: } & 1 & 3 & 1 & 3 & 1 & 3 & 1 & 3 \\ \text { Voix 4: } & 1 & 1 & 1 & 1 & 1 & 1 & 1 & 1\end{array}$

Contrairement à ce qu'on retrouvait précédemment dans l'œuvre, la durée d'une note n'est plus systématiquement issue de son numéro d'harmonique. C'est un autre processus qui est ici en jeu, un processus qui fait en sorte que toutes les durées s'acheminent graduellement vers la noire. Les durées, en nombre de triple croches, sont présentées dans le tableau suivant, qui met en lumière l'homogénéisation graduelle du rythme:

\begin{tabular}{|c|c|c|c|c|c|c|c|c|c|c|c|c|c|c|c|}
\hline \multicolumn{10}{|c|}{ VOIX $\mathbf{1}$} \\
\hline $\mathbf{1}$ & $\mathbf{3}$ & $\mathbf{5}$ & $\mathbf{7}$ & $\mathbf{9}$ & $\mathbf{1 1}$ & $\mathbf{1 3}$ & $\mathbf{1 5}$ & $\mathbf{2}$ & $\mathbf{6}$ & $\mathbf{1 0}$ & $\mathbf{1 4}$ & $\mathbf{2}$ & $\mathbf{6}$ & $\mathbf{1 0}$ & $\mathbf{1 4}$ \\
\hline 2 & 4 & 5 & 7 & 9 & 11 & 12 & 14 & $\mathbf{3}$ & $\mathbf{6}$ & $\mathbf{1 0}$ & $\mathbf{1 3}$ & $\mathbf{3}$ & $\mathbf{6}$ & $\mathbf{1 0}$ & $\mathbf{1 3}$ \\
\hline 3 & 4 & 6 & 7 & 9 & 10 & 12 & 13 & $\mathbf{4}$ & $\mathbf{7}$ & $\mathbf{9}$ & $\mathbf{1 2}$ & $\mathbf{4}$ & $\mathbf{7}$ & $\mathbf{9}$ & $\mathbf{1 2}$ \\
\hline 4 & 5 & 6 & $\mathbf{7}$ & $\mathbf{9}$ & $\mathbf{1 0}$ & $\mathbf{1 1}$ & $\mathbf{1 2}$ & $\mathbf{5}$ & $\mathbf{7}$ & $\mathbf{9}$ & $\mathbf{1 1}$ & 5 & 7 & 9 & 11 \\
\hline $\mathbf{5}$ & $\mathbf{6}$ & 7 & $\mathbf{8}$ & $\mathbf{8}$ & $\mathbf{9}$ & $\mathbf{1 0}$ & $\mathbf{1 1}$ & $\mathbf{6}$ & $\mathbf{7}$ & $\mathbf{9}$ & $\mathbf{1 0}$ & 6 & 7 & 9 & 10 \\
\hline $\mathbf{6}$ & $\mathbf{7}$ & $\mathbf{7}$ & $\mathbf{8}$ & $\mathbf{8}$ & $\mathbf{9}$ & $\mathbf{9}$ & $\mathbf{1 0}$ & $\mathbf{7}$ & $\mathbf{8}$ & $\mathbf{8}$ & $\mathbf{9}$ & 7 & 8 & 8 & 9 \\
\hline $\mathbf{7}$ & $\mathbf{7}$ & $\mathbf{8}$ & $\mathbf{8}$ & $\mathbf{8}$ & $\mathbf{8}$ & $\mathbf{9}$ & $\mathbf{9}$ & $\mathbf{8}$ & $\mathbf{8}$ & $\mathbf{8}$ & $\mathbf{8}$ & 8 & 8 & 8 & 8 \\
\hline$(\mathbf{8}$ & $\mathbf{8}$ & $\mathbf{8}$ & $\mathbf{8}$ & $\mathbf{8}$ & $\mathbf{8}$ & $\mathbf{8}$ & $\mathbf{8})$ & 8 & 8 & 8 & 8 & 8 & 8 & 8 & 8 \\
\hline
\end{tabular}

\begin{tabular}{|c|c|c|c|c|c|c|c|c|c|c|c|c|c|c|c|}
\hline \multicolumn{8}{|c|}{ VoIx 3} & \multicolumn{8}{|c|}{ VoIx 4} \\
\hline 4 & 12 & 4 & 12 & 4 & 12 & 4 & 12 & 8 & 8 & 8 & 8 & 8 & 8 & 8 & 8 \\
\hline 5 & 11 & 5 & 11 & 5 & 11 & 5 & 11 & 8 & 8 & 8 & 8 & 8 & 8 & 8 & 8 \\
\hline 6 & 10 & 6 & 10 & 6 & 10 & 6 & 10 & 8 & 8 & 8 & 8 & 8 & 8 & 8 & 8 \\
\hline 7 & 9 & 7 & 9 & 7 & 9 & 7 & 9 & 8 & 8 & 8 & 8 & 8 & 8 & 8 & 8 \\
\hline 8 & 8 & 8 & 8 & 8 & 8 & 8 & 8 & 8 & 8 & 8 & 8 & 8 & 8 & 8 & 8 \\
\hline 8 & 8 & 8 & 8 & 8 & 8 & 8 & 8 & 8 & 8 & 8 & 8 & 8 & 8 & 8 & 8 \\
\hline 8 & 8 & 8 & 8 & 8 & 8 & 8 & 8 & 8 & 8 & 8 & 8 & 8 & 8 & 8 & 8 \\
\hline 8 & 8 & 8 & 8 & 8 & 8 & 8 & 8 & 8 & 8 & 8 & 8 & 8 & 8 & 8 & 8 \\
\hline
\end{tabular}


6. Ces insertions se trouvent aux mesures 99, 116, 136 et 173 .
Dans le tableau ci-dessus, les nombres en caractères gras correspondent à des notes effectivement jouées, tandis que les autres correspondent à des notes qui ont été gommées. En effet, tandis que la voix 1 descend graduellement dans le registre de l'instrument, les trois voix inférieures se taisent l'une après l'autre.

La partie de piano est réalisée en ajoutant une doublure à chaque note de ce contrepoint virtuel. L'intervalle auquel se fait la doublure est issu des accords entendus sur la bande et change toutes les deux mesures. Ces intervalles de doublures (qui ne sont pas tempérés sur la bande mais le sont bien sûr au piano) sont les suivants, dans l'ordre: seconde majeure, quinte juste, septième majeure, dixième majeure, septième mineure, onzième juste, dixième mineure et octave juste.

\section{Les exceptions}

Tout compositeur utilisant des outils de formalisation stricts en vient un jour ou l'autre à se poser la question des exceptions. Est-il bon de briser localement ou de tordre un système compositionnel par ailleurs rigide? Pour les compositeurs dont la démarche, comme celle de Gonneville, s'inscrit dans la foulée du post-sérialisme, cette question se pose avec beaucoup de force. Face à cette question, deux types d'attitudes sont envisageables. La première attitude, qu'on pourrait qualifier de rigoriste, se résume ainsi : un bon système n'a pas besoin d'exceptions, ce qui implique qu'un système qu'on sent le besoin de briser, même localement, est un mauvais système. Les tenants de cette position sont à la recherche d'une perfection mécanique. La seconde attitude, empreinte de plus de souplesse, repose sur l'idée selon laquelle un système n'est pas une fin en soi, qu'il s'agit d'un outil comme un autre pour atteindre certains objectifs musicaux. Dans cette optique, toute exception permettant d'atteindre plus efficacement les objectifs fixés est permise. Les tenants de cette position sont à la recherche d'une beauté organique. Il semble que Gonneville doive être associé à cette deuxième attitude. Pour nous en convaincre, examinons maintenant quelques exceptions aux systèmes mis en œuvre dans Chute/Parachute.

Le type d'exception le plus fréquent dans l'œuvre est l'insertion. Comme nous l'avons vu, le rythme est généralement encadré par des systèmes compositionnels assez rigides. Ceci fait en sorte que tout élément ajouté à la structure sera assez visible. Par exemple, entre les cinq sections de la partie B, une mesure a systématiquement été ajoutée à la structure en guise de transition ${ }^{6}$. Ces mesures insérées font office de point d'orgue et signalent le changement de section tout en préparant l'oreille pour le matériau mélodique à venir. 
Toujours dans la partie $B$, dans la quatrième section, on retrouve sept insertions?. Ces insertions agissent encore une fois comme des points d'orgue qui interrompent momentanément le contrepoint à quatre voix qui constitue le système principal. Dans ce cas-ci, ces insertions mêmes ont été, en quelque sorte, formalisées, puisqu'elles ont été conçues de façon très systématique. La durée de ces insertions va en décroissant tandis que leur intensité croît. Les hauteurs qu'elles font entendre sont également liées de façon systématique au système harmonique du contrepoint qu'elles interrompent. Ces sept insertions ont ceci de particulier qu'elles sont si prédominantes sur le plan de la perception que ce sont elles qui constituent le principal élément de directionnalité de la section; elles créent un accroissement graduel de la tension qui mènera à la dernière section de $\mathrm{B}$, que nous avons examinée plus haut. Perceptuellement, donc, les exceptions sont ici plus importantes que le système, bien que ces exceptions soient elles-mêmes l'expression d'un autre système.

Signalons finalement une exception plus subtile qui se trouve à la dernière mesure de l'œuvre. La bande y présente un arpège d'harmoniques impairs. Or, cet arpège se résout exceptionnellement sur un harmonique pair, le do aigu, afin de donner une impression de résolution, le do tenant le rôle et étant effectivement perçu comme la fondamentale de toute l'œuvre. Le compositeur a sans doute senti la nécessité de résoudre l'œuvre, d'où cette exception, qui n'avait aucune nécessité sur le plan structurel. Cette résolution est d'ailleurs accentuée par le glissando final, à partir du do, vers le suraigu inaudible.

\section{Structure et perception}

Ce bref survol analytique de Chute/Parachute nous permet de constater une part de la grande originalité de l'attitude de Gonneville vis-à-vis de la notion de structure. Car la nature provocatrice et la contemporanéité aiguë de la démarche de Gonneville tiennent beaucoup au fait qu'il utilise des techniques compositionnelles de type sériel exclusivement en fonction de leur impact sur la perception. Gonneville est à la recherche d'une musique qui dise et qui fasse sentir plutôt que d'une musique qui montre, objectif naturel de la démarche sérielle. En somme, Gonneville met la structure entièrement au service de la perception et de l'expression, et sa réintégration de la périodicité et de la consonance au sein du système de pensée sériel est emblématique de cette volonté.

Un rapprochement doit également être fait avec la démarche de Stockhausen, et notamment avec son concept de «formule». Le travail des
7. Ces insertions se retrouvent aux mesures 138 à 142, 144 à 147, 149 à 151 , 154 à $156,160-161,165$ et 170 
formules, chez Stockhausen, vise justement à donner une existence sonore aux structures, à faire entendre les structures. On pourrait, quoique dans une moindre mesure, dire la même chose de l'approche sérialiste intégrale, qui cherche, par exemple, à faire entendre rythmiquement les hauteurs et à faire entendre mélodiquement et harmoniquement les rythmes. Gonneville, qui a étudié auprès de Stockhausen durant les années 1970, dit avoir pris chez le compositeur allemand certaines techniques qui ont servi de base à l'élaboration de son propre langage musical. Le travail sur les nombres et la séparation des paramètres musicaux font partie de ces techniques. L'influence de Stockhausen est également sensible sur le plan du travail formel. Comme Stockhausen, Gonneville a développé un sens aigu de l'à-propos formel: les formes qu'il emploie donnent une impression de solidité et soutiennent constamment l'intérêt par des pauses, des climax et des parenthèses de différents types. Stockhausen est un compositeur qui a toujours cherché à dépasser les tabous, à abattre les frontières. Gonneville, qui partage tout à fait cette attitude, a pris le flambeau de la pensée sérielle la plus intransigeante et lui a ouvert de nouvelles voies, en abattant les frontières stylistiques à l'intérieur desquelles elle avait été jusqu'alors confinée.

\section{B I B L I O G RA P H IE}

Auster, Paul (1993), Léviathan, traduction: C. Le Bœuf, Arles, Actes Sud, Coll. «Babel». zOLA, Émile (2000), L'Assommoir (1877), Paris, Flammarion. 